\title{
Poser clairement la question des interfaces
}

\section{Bruno Kesseli}

Dr med. et lic. phil., rédacteur en chef

Lédition 2016 de la désormais traditionnelle Journée de réflexion de l'Institut suisse pour la formation médicale postgraduée et continue (ISFM) et du Collège des Doyens des facultés de médecine suisses a offert un mélange passionnant d'informations, d'idées et d'exposés sur la formation médicale prégraduée, postgraduée et continue.

"Ils sont quasiment tous là», a relevé le président de l'ISFM Werner Bauer dans son discours d'ouverture à la Journée de réflexion 2016. Force est de constater qu'il dit vrai, puisque les représentants renommés d'un très grand nombre d'institutions et d'organisations impliquées dans la formation des médecins ont répondu présent. Fidèle à son nom, la Journée de réflexion souhaite non seulement accorder une large place à l'échange d'idées, à la réflexion et au débat, comme Werner Bauer l'a d'emblée souligné, mais également permettre de poser clairement la question des interfaces. Werner Bauer a ainsi anticipé ce qui allait se dire tout au long de la journée, à savoir que la formation, qu'elle soit prégraduée, postgraduée ou continue, ne doit pas être un bloc fermé et isolé, mais s'intégrer dans un ensemble continu où chaque étape permet d'acquérir des compétences sur lesquelles reposent celles acquises lors de la suivante.

Contrairement aux Journées de réflexion précédentes, celle-ci ne s'est pas déroulée à Brunnen comme à l'accoutumée, mais à Lucerne, pour raccourcir quelque peu le trajet des collègues romands, comme l'a fait remarquer avec humour Henri Bounameaux, doyen de la Faculté de médecine de l'Université de Genève, président du Collège des Doyens, mais aussi codirecteur et co-animateur de cette édition 2016.

\section{Après le «SCLO», voici «PROFILES»}

Pierre-André Michaud de la Commission interfacultés médicale suisse (CIMS) a ouvert la danse avec le premier bloc thématique sur «Les étapes de la formation

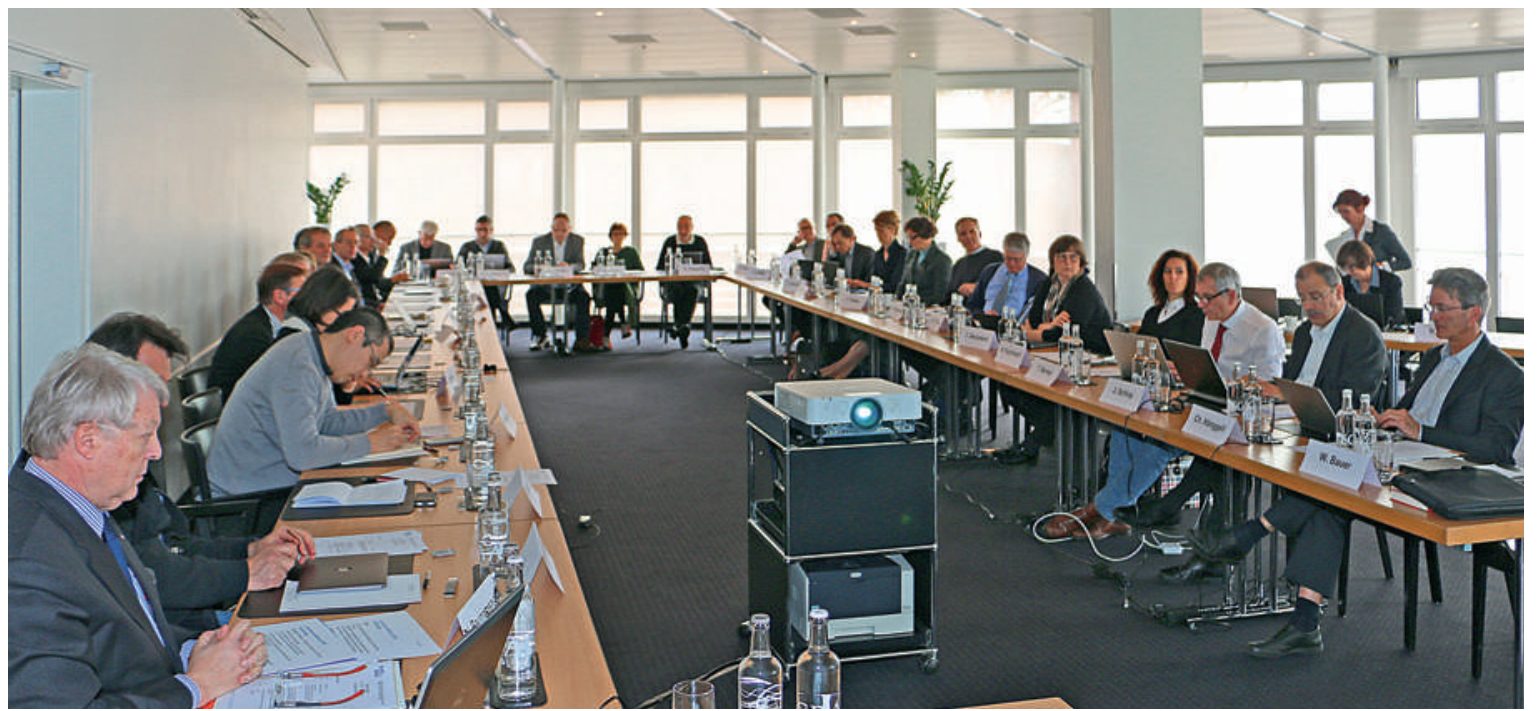

Comme son nom l'indique, la «Journée de réflexion» permet aux experts des différents domaines de la formation médicale d'échanger leurs points de vue. 


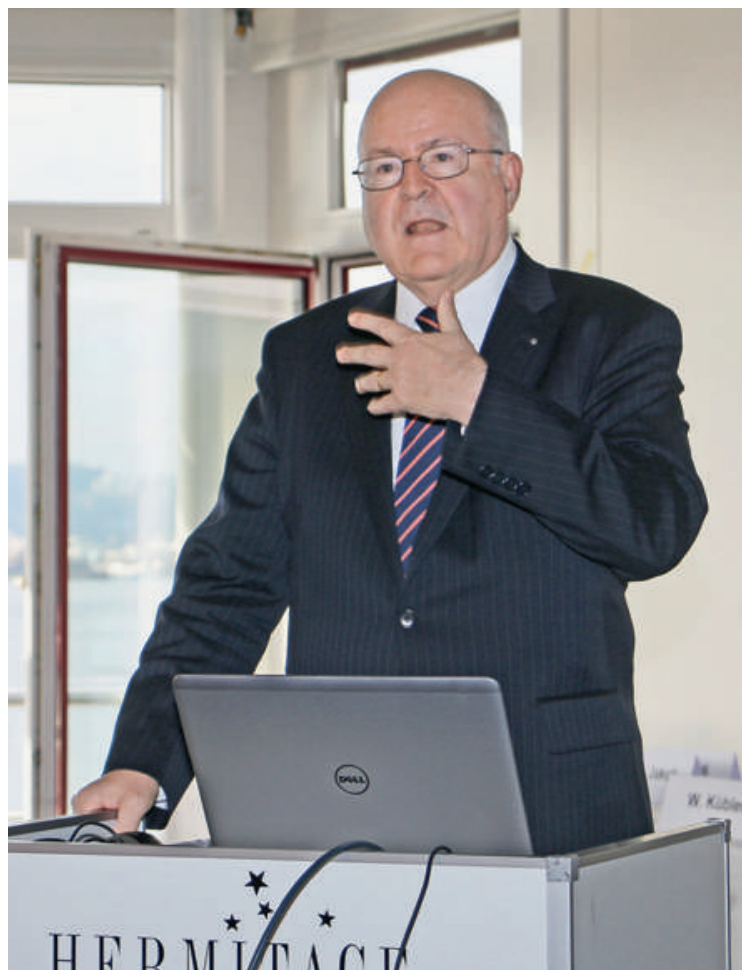

"lls sont tous là»: Werner Bauer, président de I'ISFM, ouvre la séance.

médicale et leur futur développement» et a présenté le nouveau catalogue des objectifs de formation PROFILES pour «Principal Relevant Objectives and Framework for Integrated Learning and Education in Switzerland». Les PROFILES remplacent le «Swiss Catalogue of Learning Objectives» (SCLO) des étudiants en médecine. En privilégiant une approche fondée sur les compétences, ces objectifs visent à encourager la flexibilité et l'autonomie des étudiants et à améliorer leur raisonnement clinique ("Clinical reasoning») afin de former des médecins qui, au terme de leurs études, seront opérationnels dès leur premier jour d'assistanat dans un établissement de formation postgraduée. Dans ce but, les EPA ou «Entrustable Professional Activities» jouent un rôle clé. Par EPA, on entend les compétences professionnelles dont la maîtrise est telle qu'elles peuvent être fournies sans supervision. Les EPA intègrent différentes compétences et disciplines et s'acquièrent par étapes progressives au cours des études. Grâce à eux, les médecins-assistants devraient dès leur premier poste être en mesure de gérer correctement les 20 à 30 premières minutes d'une situation d'urgence.

Même si tous les participants connaissent le concept des PROFILES, l'exposé de Pierre-André Michaud a quand même apporté de nouveaux éléments et suscité un débat animé sur différentes questions. Peter Meier$A b t$, président de l'Académie suisse des sciences médicales (ASSM), a souhaité savoir si ce nouveau modèle permettait aux étudiants de se familiariser avec l'EBM*, le "Big Data», la médecine personnalisée, et d'autres sujets qui gagnent en importance. Reto Meuli, directeur de l'Ecole de formation postgraduée de la Faculté de médecine de Lausanne a demandé si l'acquisition de compétences dans le domaine de l'éthique et de la politique de la santé faisait également partie du programme. Pierre-André Michaud a répondu par l'affirmative dans les deux cas en précisant qu'il étaitprévu de mettre sur pied une plateforme interactive pour pouvoir adapter les PROFILES. Suivront une consultation des facultés de médecine, une adaptation en association avec différentes commissions concernées et enfin l'approbation du projet par la CIMS sous «l'égide de l'OFSP», également partenaire du projet. La phase initiale d'implémentation des PROFILES est prévue pour 2017.

\section{La formation postgraduée en période de changements structurels}

Werner Kübler, directeur de l'Hôpital universitaire de Bâle, a illustré dans son exposé "Contraintes hospitalières et formation des médecins" de nombreux exemples pour mettre en évidence l'impact des changements structurels sur la formation postgraduée des médecins. Les hôpitaux sont aujourd'hui confrontés à de nombreux défis tant économiques qu'organisationnels qui ne sont pas toujours compatibles avec les modèles de formation actuels. Dans ce contexte, Werner Kübler a notamment évoqué la création de centres interdisciplinaires, la coopération plurielle avec des «mé-

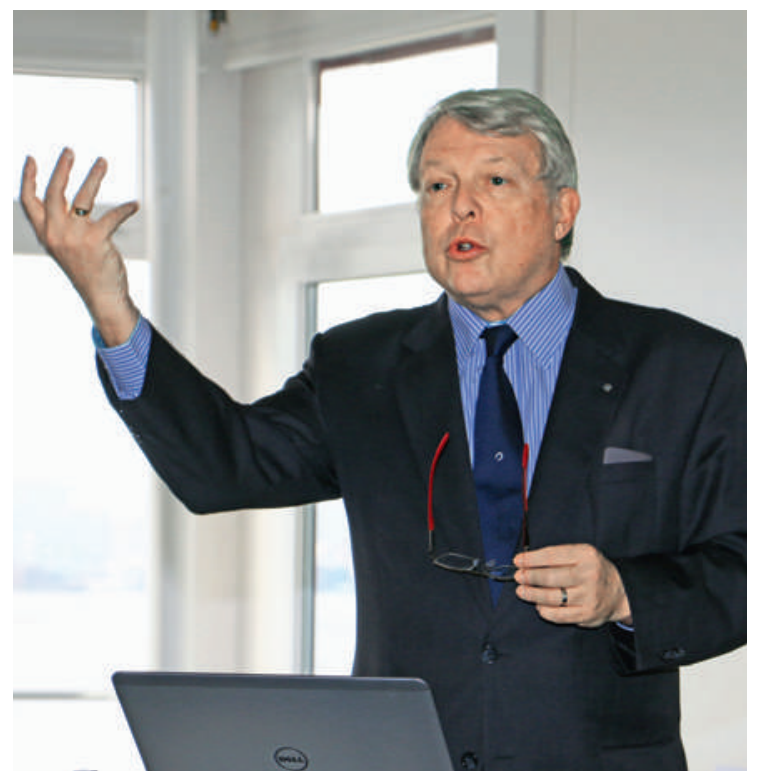

Co-organisateur et co-animateur: Henri Bounameaux, président du Collège des Doyens. 


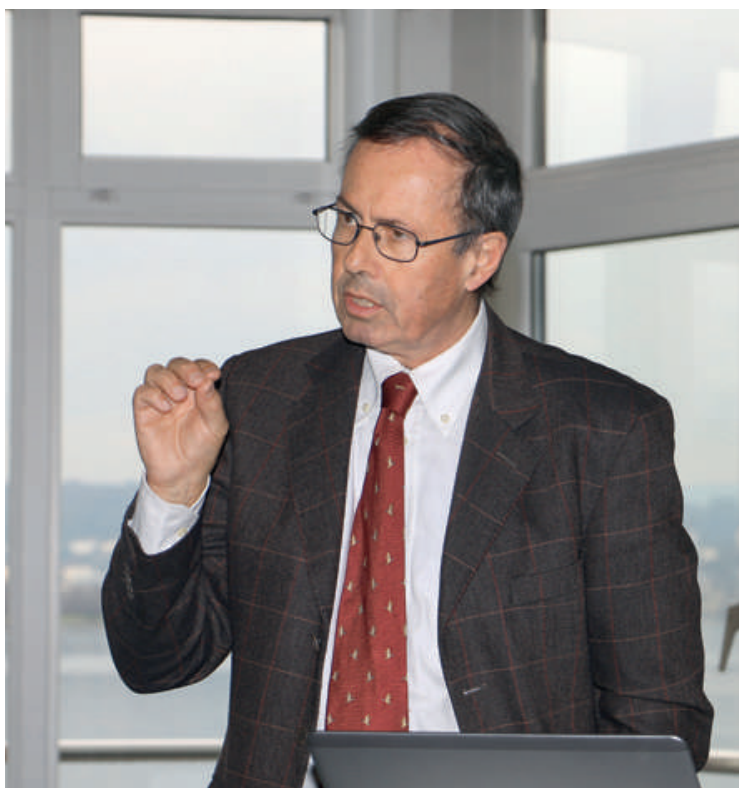

Pierre-André Michaud a présenté le nouveau catalogue des objectifs de formation "PROFILES".

decins mobiles» ou, dans le sillage d'une médecine personnalisée, le passage des cliniques spécialisées aux équipes interprofessionnelles. Ces évolutions sont en partie en contradiction avec les exigences requises en matière de structure et d'organisation en vue de la reconnaissance des établissements de formation postgraduée, et cela crée, selon Kübler, une «structure instable». Ces évolutions comportent cependant aussi des avantages. Il cite à titre d'exemple extrêmement positif le réseau de formation postgraduée en médecine de famille pour la Suisse du Nord-Est. Selon lui, on assiste à un basculement: on dépasse l'établissement de formation en tant que structure pour aller au contenu de la formation, autrement dit, on va au-delà du lieu de formation caractérisé par l'établissement de formation pour privilégier le réseau de formation postgraduée. L'importance des compétences médicales interdisciplinaires va probablement augmenter, et une formation postgraduée axée principalement sur la spécialisation va certainement évoluer dans le sens d'une "personnalité professionnelle». Par ailleurs, la discussion a confirmé la nécessité de répondre aux nouveaux problèmes d'interface et de structure par des solutions flexibles et modulaires basées sur la mise en réseaux. L'ISFM estime que c'est tout à fait envisable si la discussion à ce sujet est lancée suffisamment tôt.

\section{"Bienvenue en Suisse»}

Le chef du groupe PLR et conseiller national Ignazio Cassis a montré qu'on pouvait être médecin et réussir brillamment en politique. Lors de la Journée de ré- flexion, le Tessinois s'est prêté au jeu des questionsréponses avec Werner Bauer et le public. Pour lui, la représentation selon laquelle un médecin fait obligatoirement du lobbying en faveur du corps médical est une «idée romantique». Il se voit plutôt comme un ardent défenseur des valeurs libérales. Il a expliqué ce que cela signifiait avec une désinvolture peu commune pour un politicien. Lorsqu'il a pointé du doigt l'absence de politique nationale en matière de santé, on lui a rétorqué sèchement: «Bienvenue en Suisse!». Une politique nationale faible dans ce domaine est une des caractéristiques du fédéralisme. A la question de savoir comment régler l'«anarchie» qui règne au sein du système de santé, il a répondu que notre système de santé n'avait rien d'anarchique, mais qu'il était au contraire hyper régulé. Pour les libéraux, la solution consisterait en un marché régulé où les partenaires tarifaires négocieraient les prix. Or ce n'est pas le cas aujourd'hui ou seulement de manière très restreinte. La bureaucratisation et la surrégulation ont créé de nombreux problèmes, et les nombreuses ordonnances fédérales ont un effet paralysant. Ignazio Cassis a admis après une intervention de Nadine Facchinetti qu'on ne pouvait pas accuser l'administration fédérale de tous les maux. La responsable par intérim du département Professions de la santé de l'Office fédéral de la santé publique avait en effet rappelé que dans le cas de la loi sur les professions médicales, c'est le Parlement qui avait gonflé une ordonnance initialement «digeste».

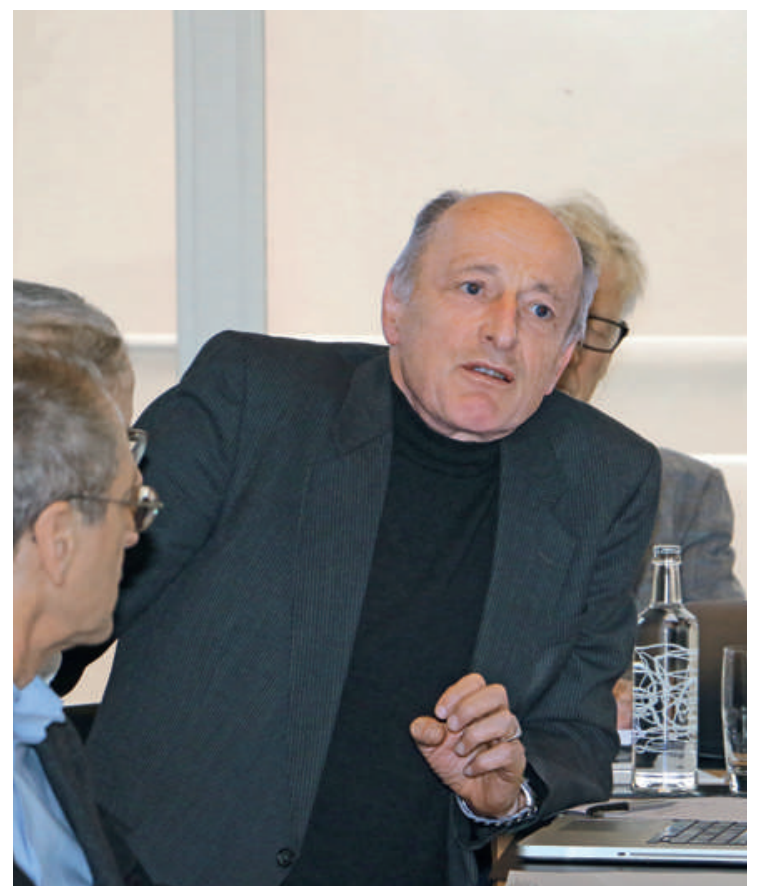

«EBM», «Big Data» et «Médecine personnalisée»: Peter Meier-Abt, président de I'ASSM. 


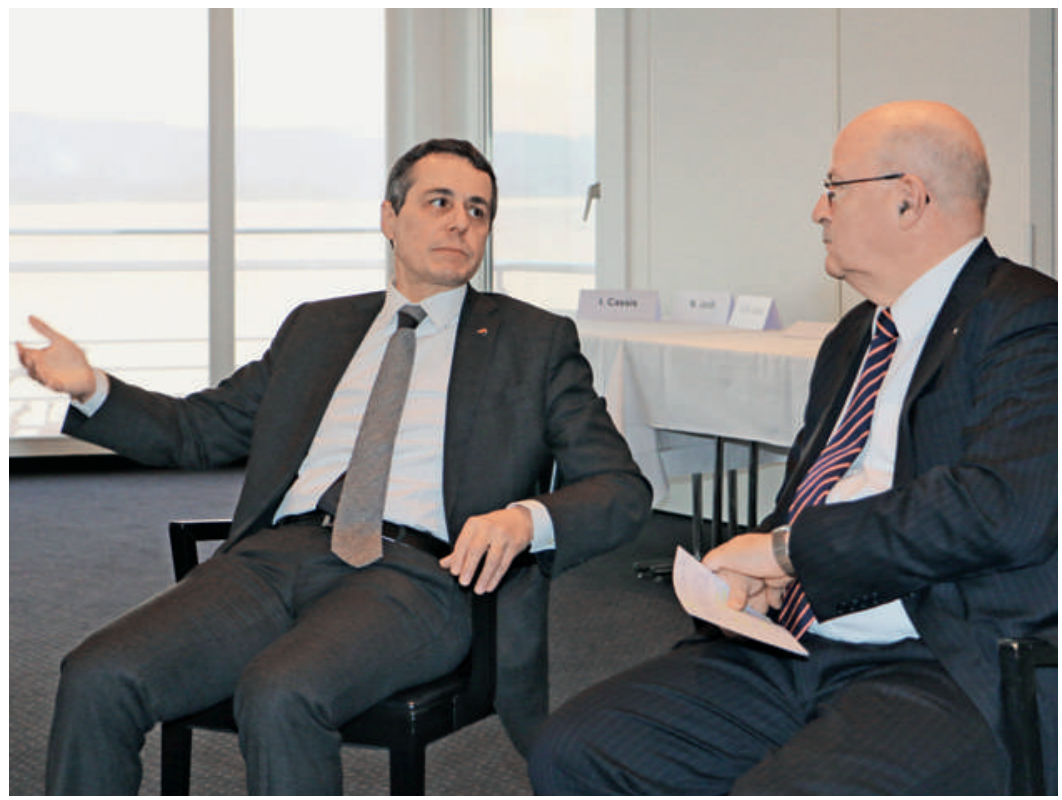

Lobbyiste des valeurs libérales: Ignazio Cassis (à gauche) en discussion avec Werner Bauer. temps l'objectif de la politique universitaire en Allemagne.»

On ne saurait reprocher à Josef Pfeilschifter de ne pas savoir de quoi il parle, lui qui a été membre de la commission à l'origine du «Catalogue national des objectifs de formation basés sur les compétences en médecine» (NKLM) . A ce moment-là, il s'est surtout agit pour lui «d'éviter les conflits» et de souligner «le rôle de la science et du savoir dans la formation». J. Pfeilschifter s'est en effet montré très sceptique envers la tendance également présente en Allemagne d'axer la formation uniquement sur les compétences. Selon lui, la compétence à disposer du savoir ne suffit pas, car «celui qui pourrait apprendre à lire, mais ne sait pas lire, demeure analphabète». Pour pouvoir apprendre et emmagasiner des connaissances, il faut déjà savoir. Les aptitudes aussi sont une forme de connaissance, de la connaissance procédurale.

J. Pfeilschifter a défendu la fameuse "connaissance cristalline» des données et des faits, car elle est indispensable aux associations créatives et à la cognition elle-même. Un «canon du savoir» est un moment d'intégration clé pour toute communauté. Or nous risquons de perdre ce cadre de référence socioculturel commun et il se pourrait que ce soit voulu. Nous préparons le terrain "pour les chasseurs de rats, les croisés, les djihadistes, les homéopathes, les faiseurs de pluie et de beau temps de tous bords qui ne demandent qu'à combler ces lacunes en matière de connaissance par leur bavardage normatif». Entre-temps, on a exigé des universités qu'elles mettent en place des «safe spaces

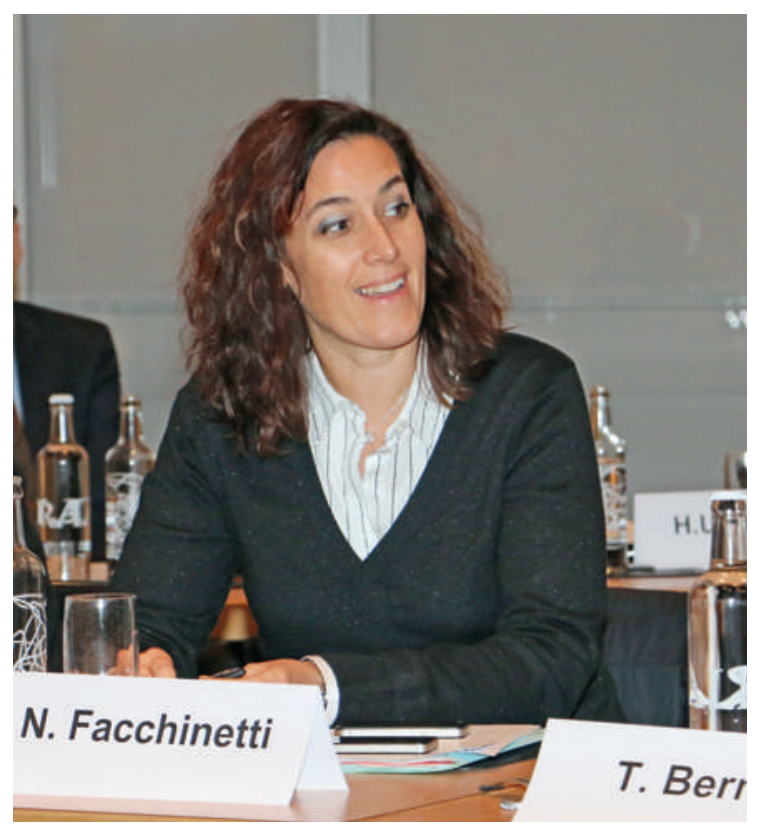

La tendance à la bureaucratie se retrouve aussi au Parlement: Nadine Facchinetti, représentante de I'OFSP. 


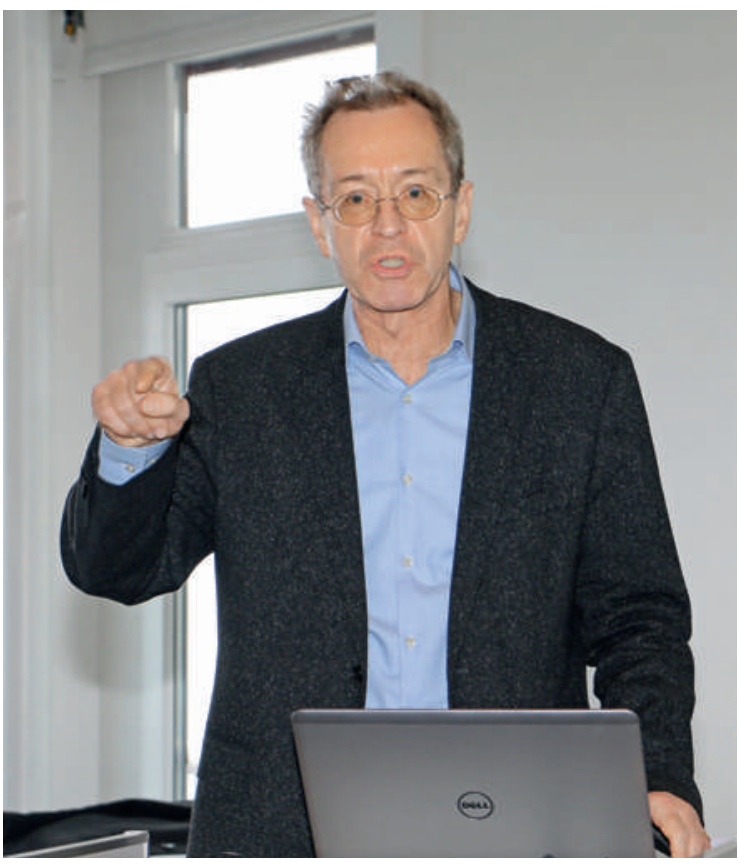

Plaidoyer pour le savoir et la science: Josef Pfeilschifter.

du non-savoir». "La connaissance dérange», selon J. Pfeilschifter, et "celui qui peut déterminer quelle connaissance dérange détient le pouvoir».

Cela s'applique particulièrement au domaine scientifique, où la «didactique de résolution de problèmes basée sur les compétences» est vouée à l'échec, car la science est par définition un processus avec des résultats ouverts. Nous «nous trahirions nous-mêmes si nous cachions cela à nos étudiants». Il termine son exposé par le constat que la science peut aussi être amusante.

\section{Les questions en suspens de la formation des médecins}

Suite à la présentation par Henri Bounameaux, en qualité de président, de la prise de position du Collège des Doyens sur la formation des médecins [2], le second bloc thématique a porté sur les questions en suspens de la formation médicale en Suisse qui sont nombreuses. Sans surprise, la question du financement de la formation postgraduée a une nouvelle fois été abor- dée. Werner Bauer a saisi l'occasion pour demander à Michael Jordi, secrétaire central de la Conférence des directeurs cantonaux de la santé, si l'ISFM pouvait contribuer à résoudre ce problème. Michael Jordi lui a recommandé de s'adresser aux départements de la santé des différents cantons via les sociétés cantonales de médecine. De telles interventions pourraient porter leurs fruits dans les cantons qui prennent des mesures d'économie.

Il a également été question de l'optimisation de la collaboration entre les facultés de médecine, les hôpitaux universitaires et l'ISFM, de la définition des objectifs de formation, et de l'éventuelle régulation des cursus de formation en Suisse. En ce qui concerne ce dernier point, certains participants ont pu compléter leur liste de travaux à faire: les facultés de médecine auront à examiner dans quelle mesure et de quelle manière elles vont unifier leurs exigences pour l'obtention d'un doctorat, et il reviendra à l'ISFM de clarifier la question de savoir s'il est possible de réduire le nombre de publications requises pour l'obtention d'un titre de spécialiste.

Pour clore la journée, Werner Bauer a une nouvelle fois cité Sir William Osler**, dont le message réjouira certainement ceux qui s'inquiètent de la qualité de la formation médicale en raison de problèmes non résolus. La remarque d'Osler publiée en 1909 dans la revue JAMA incite à l'optimisme: "A faculty without its troubles is always in a bad way - the water should be stirred. Some ferment should be brewing; the young men should always be asking for improvements, to which the old men would object.»

\section{Crédit photos \\ Bruno Kesseli}

\section{Références}

1 Vous trouverez de plus amples informations sur les sujets précités dans les articles suivants: Kesseli B. Actif sur plusieurs terrains. Assemblée plénière de l'ISFM du 26 novembre 2015 à Berne. Bulletin des médecins suisses. 2016;97(4):124-8.

Hänggeli C, Kuhn HP, Linder B. Mention des titres facilitée. Bulletin des médecins suisses. 2015;96(50-51):1834-6.

2 Dans l'intervalle, ce document a aussi été publié dans le BMS: Bounameaux H, Eggli P, Gasser T, Tissot JD, Weber R. Statement regarding medical education in Switzerland. Bulletin des médecins suisses. 2016;97(12-13):461-2. 